\title{
Evaporation of Micro-Droplets: the "Radius-Square-Law" Revisited
}

\author{
D. JakubczyK*, M. Kolwas, G. Derkachov, K. Kolwas and M. Zientara \\ Institute of Physics, Polish Academy of Sciences, al. Lotników 32/46, 02-668 Warsaw, Poland
}

(Received August 21, 2012)

\begin{abstract}
The range of applicability of a fundamental tool for studying the evolution of droplets, the "radius-square-law", was investigated both analytically and numerically, on the basis of the experimental results of our own as well as of other authors. Standard issues were briefly discussed. Departures from the "radius-square-law" caused by the influence of impurities encountered in non-ideal liquids, by the kinetic and surface tension effects encountered for small droplets or by thermal imbalance encountered in light-absorbing droplets were analysed. The entanglement between the kinetic and impurities effects was studied numerically yielding a possible explanation to evaporation coefficient discrepancies found in the literature. An unexpected "radius-square-law" persistence in case of non-isothermal evolutions of very small droplets in atmosphere nearly saturated with vapour was analysed. The coexistence of the kinetic effects and the strong effects of surface tension was found responsible for this effect.
\end{abstract}

PACS: 64.70.F-, 64.70.fm, 68.03.Fg, 68.03.Hj, 05.70.Ln

\section{Introduction}

The ubiquitous processes of evaporation and condensation are still not fully understood and the awareness of that fact seems to increase [1,2]. The continued interest in detailed modelling of these processes, especially concerning droplets, is strongly driven by their role in the Earth ecosystem, as well as their indispensability in technology. However, there is also a more fundamental aspect of making the theories consistent. The continuous-medium descriptions of evaporation/condensation phenomena (see e.g. $[3,4]$ ) cannot grasp the details at molecular level, so far (compare e.g. [5]). On the other hand, though it seems possible to learn a lot with molecular dynamics (MD) simulations (see e.g. $[6,7]$ ), the large scale, engineering applications of MD is far from feasible. A new attitude is often sought. The application of statistical rate theory (SRT) [8, 9] or irreversible thermodynamics $[10,11]$ make an example.

However, in wait for the scientific breakthrough, one resorts to currently available methods. It turns out that they can provide many valuable information, if they are carefully applied. As far as the evaporation/condensation of droplets is concerned the famous "radius-square-law" is the fundamental tool ("diameter-square-law" is also met in the literature). This law states that the square of droplet radius tends to evolve linearly in time. In other words, there is a conservation law for the evaporation/ condensation of droplets: the rate at which the surface area changes is constant. The "radius-square-law" reflects

* corresponding author; e-mail: jakub@ifpan.edu.pl also an every-day-life intuition that the effectiveness of drying depends on the surface area of the dried object. In this work we study the range of applicability of this law and its extensions to droplets of non-ideal liquids. Apart from the evolutions which adhere to the "radius-square-law" quite obviously, we analyse cases which conform to the "radius-square-law" rather unexpectedly, as well as seemingly similar cases in which we encounter evident departures from the "radius-square-law". We refer to the results of our own experiments (the datasets presented here have not been published yet), as well as to the experimental results of other researchers. The description of our experimental methods can be found in [12-14] and a short summary is given in the next section.

\section{Experimental setup and procedures}

The single droplets that we studied, were levitated in an electrodynamic quadrupole trap (compare e.g. [15-19]), a variant of which we developed in our lab. The trap was kept in a small $\left(\approx 10 \mathrm{~cm}^{3}\right)$ thermostatic chamber. Droplets were injected into the trap with the droplet-on-demand injector (compare e.g. [20, 21]), a variant of which we developed. The injector was kept inside the chamber ensuring that the initial temperature of the droplet was equal to that of the chamber. On emerging from the injector nozzle, the droplets were charged by charge separation in the external field of the trap. The experimental setup schematic view is presented in Fig. 1.

In experiments presented in this work we used: (i) ultrapure water produced in the lab (Milli-Q Plus, Millipore, resistivity $\approx 18 \mathrm{M} \Omega \mathrm{cm}$, total dissolved solids 


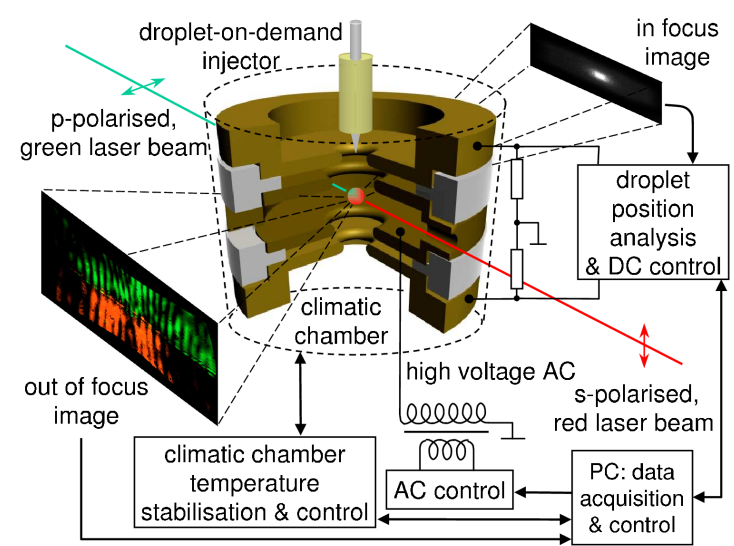

Fig. 1. Experimental setup schematic view.

$<20 \mathrm{ppb}$, total organic carbon $\leq 10 \mathrm{ppb}$, no suspended particles larger than $0.22 \mu \mathrm{m}$, microorganisms $\leq 1$ colony forming unit per ml, silicates $<0.1 \mathrm{ppb}$ and heavy metals $\leq 1 \mathrm{ppb}$ ), (ii) diethylene glycol (Fluka, BioUltra, 99.99 GC area \%), (iii) triethylene glycol (Fluka, BioUltra, anhydrous, $99.96 \mathrm{GC}$ area \%), (iv) dimethyl sulfoxide (Sigma-Aldrich, A.C.S. spectrophotometric grade, 99.99 GC area \%) and (v) $\mathrm{C}_{60}$ nanocrystallites water suspension. The suspension was prepared by sonicating fullerene powder $\left(99 \% \mathrm{C}_{60}, \mathrm{MER}\right)$ in ultrapure water and filtering through a filtering paper $(\approx 15 \mu \mathrm{m}$ pore size) and $450 \mathrm{~nm}$ pore size filter (SM11306, Sartorius) shortly before the experiment. In case of experiments with water and with $\mathrm{C}_{60}$ /water suspension droplets, the chamber was filled with water vapor/nitrogen mixture at atmospheric pressure. In case of other liquids that we studied, there was no intentional admixture of water vapour to dry nitrogen.

The temporal evolution of the droplet radius $a(t)$, was obtained by analysing the angular distribution of scattered light irradiance (angle-resolved static light scattering, see eg. [22-24] and references therein). In case of pure liquids, exact Mie theory predictions were fitted to the experimentally obtained scattering patterns, yielding droplet radius with high precision (up to $\pm 10 \mathrm{~nm}$ for slowly evaporating liquids). In case of droplets of suspension, an approximate $( \pm 150 \mathrm{~nm})$ but more robust method, directly linking the droplet radius with the characteristic angular frequency of scattering pattern was used. Two coaxial, counter propagating laser beams were used simultaneously for droplet illumination: green $(532.07 \mathrm{~nm}) p$-polarised and red $(654.25 \mathrm{~nm}) s$-polarised. Droplet heating and direct momentum transfer from the beams were negligible.

\section{Basic dynamics of droplet evolution}

The problem of stationary evaporation of a free, spherical, motionless droplet of a pure liquid in an infinite, inert medium was first addressed by Maxwell (wet-bulb thermometer [25]). Despite known shortcomings of
Maxwellian description [26, 2], evaporation (or growth) models based on it are still widely utilised and considered generally adequate. Though originally Maxwell derived his formulae simply from Fick's first law, they can be further traced to more general equations of fluid dynamics and, ultimately, to the Boltzmann equation (see e.g. $[27,3])$.

The framework of Maxwellian description consists of a set of equations for the transport of mass and heat. The mass transport equation usually attracts attention first, since the mass of a micron-sized droplet is experimentally accessible with high accuracy (via radius measurement), while the temperature of such droplet only with rather limited. The equation of the transport of mass can be written in a form binding the droplet surface area $\left(4 \pi a^{2}\right)$ and the vapour density difference between the vicinity of the surface $\rho_{\text {sur }}$ and the reservoir (climatic chamber (cc)) $\rho_{\mathrm{cc}}$ :

$$
\frac{\mathrm{d} a^{2}}{\mathrm{~d} t}=2 \frac{D_{k}}{\rho_{\mathrm{L}}}\left[\rho_{\mathrm{cc}}\left(T_{\mathrm{cc}}\right)-\rho_{\mathrm{sur}}\left(T_{\mathrm{L}}\right)\right],
$$

where $\rho_{\mathrm{L}}$ is the density of the liquid and $T_{\mathrm{L}}$ and $T_{\mathrm{cc}}$ are the temperature of the droplet (surface) and the climatic chamber respectively. Since the language of diffusive transport is not suitable for sub-micron distances, the above equation is generalised by the use of the effective diffusion coefficient $D_{k}$, effective for sum-micron-sized droplets. This coefficient encompasses, so-called, gas kinetic correction, which will be discussed in Sect. 5.1. For very small droplets or very small $\rho_{\mathrm{cc}}\left(T_{\mathrm{cc}}\right)-\rho_{\mathrm{sur}}\left(T_{\mathrm{L}}\right)$ using the Kelvin equation is necessary

$$
\rho_{\text {sur }}\left(T_{\mathrm{L}}\right)=\rho_{\text {sat }}\left(T_{\mathrm{L}}\right) \exp \left(\frac{2 M \sigma}{R T_{\mathrm{L}} \rho_{\mathrm{L}} a}\right),
$$

where $\sigma$ is the surface tension of the liquid.

In general, the transfer of heat to/from the droplet should be considered simultaneously with the transport of mass. Under the assumption that evaporation/condensation is associated only with the heat transported via conduction between the droplet and the reservoir ("instantaneous evaporation"), a simple formula [4] is obtained:

$$
\Delta T=\frac{h_{\mathrm{LV}} \rho_{\mathrm{L}}}{2 \lambda_{k}} \frac{\mathrm{d} a^{2}}{\mathrm{~d} t},
$$

where $\Delta T=T_{\mathrm{L}}-T_{\mathrm{cc}}, h_{\mathrm{LV}}$ is the (effective, see Sect. 6) enthalpy of evaporation and $\lambda_{k}$ is the effective thermal conductivity (kinetic effects accounted for) of the ambient gas-vapour mixture. The above assumption seems well justified for the stationary evolution of a small droplet (compare [28, 29]), and is readily used.

\section{The "radius-square-law" for isothermal evolution}

It can be easily demonstrated that the "radius-square-law" follows from the Maxwellian framework as a first approximation. If there are no kinetic effects $\left(D_{k}=D\right.$ and $\lambda_{k}=\lambda$, see Sect. 5.1), no surface tension effects (vapour far from saturation: $\rho_{\mathrm{cc}}-\rho_{\mathrm{sur}} \gg 0$, large 
droplets) and (nearly) isothermal evolution $\left(T_{\mathrm{L}} \approx\right.$ const, $\rho_{\text {sur }}=$ const) Eq. (1) yields the "radius-square-law":

$$
a^{2}(t)-a^{2}(t=0)=A t,
$$

where

$$
A=2 \frac{D}{\rho_{\mathrm{L}}}\left(\rho_{\mathrm{cc}}-\rho_{\mathrm{sur}}\right) .
$$

Obviously, $\Delta T=$ const consistently follows. Such mode of evolution is illustrated with two examples in Fig. 2. Both graphs concern relatively large droplets and mod-

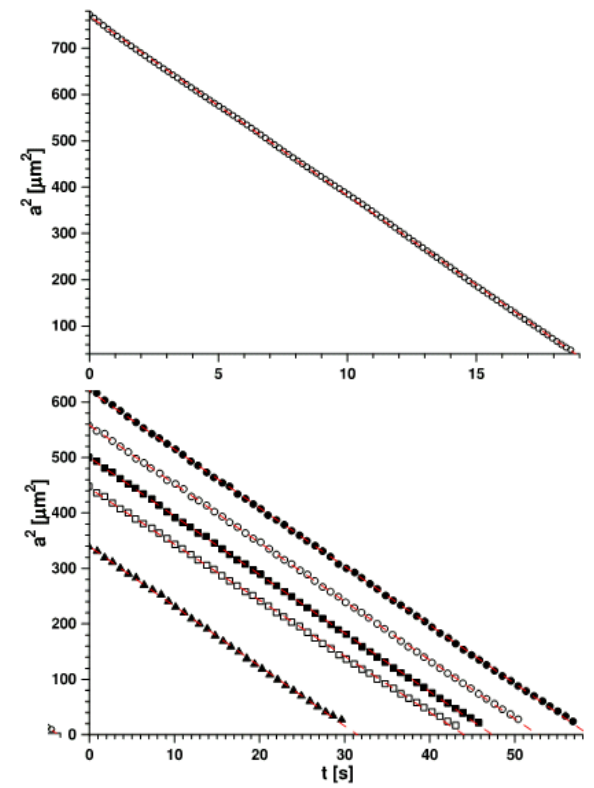

Fig. 2. Temporal evolution of the square of the radius of evaporating droplet(s). Upper part: dimethyl sulfoxide (DMSO) in $\mathrm{N}_{2}$ atmosphere initially void of DMSO vapour $\left(\rho_{\mathrm{cc}}=0\right), T_{\mathrm{cc}}=298 \mathrm{~K}, 1004 \mathrm{hPa}$ pressure. Data obtained from our experiment, every second point displayed. Lower part: water in wet (dew point at $229.62 \mathrm{~K}) \mathrm{N}_{2}$ atmosphere, $T_{\mathrm{cc}}=239.75 \mathrm{~K}, 593 \mathrm{hPa}$ pressure. Data from [30]. Dashed (red) lines correspond to linear fits - the "radius-square-law".

erate rate of evaporation (small $\Delta T$ ). The upper graph visualises an evolution corresponding to $\rho_{\mathrm{cc}}=0$ (surrounding gas initially void of vapour), exemplified by evaporation of a droplet of dimethyl sulfoxide (DMSO), obtained from our experiments. The lower graph visualises an evolution in gas-vapour mixture, exemplified by evaporation of water droplets in wet nitrogen atmosphere (moderately hight humidity), obtained from [30].

\section{Departures from the "radius-square-law"}

Though the "radius-square-law" seems to be a quite robust tool, the departures are quite often encountered. Here we shall discuss three classes of phenomena which introduce non-linearity to $a^{2}(t)$ evolution: kinetic effects, influence of impurities and additional heat. We shall also study a case of seemingly unexpected adherence to the "radius-square-law".

\subsection{Kinetic effects}

The equations of fluid dynamics do not hold where the gradients of described quantities are high. Such is exactly the case in the vicinity of gas-liquid interface, below the mean free path of the gas molecule from the surface. Customarily, however not with full exactness (see $[31,4,32,2,13]$ ), the transport of molecules in this region is treated as ballistic and thus governed by the Hertz-Knudsen-Langmuir (HKL) equation.

For a droplet in vacuum the mass transport equation derived from kinetic theory of gases takes the following form:

$$
\frac{\mathrm{d} a}{\mathrm{~d} t}=-\frac{\alpha_{\mathrm{C}}}{\rho_{\mathrm{L}}} v\left(T_{\mathrm{L}}\right) \rho_{\mathrm{sur}}\left(T_{\mathrm{L}}\right),
$$

where $v$ is average thermal velocity of vapour molecules and $\alpha_{\mathrm{C}}$ is the evaporation coefficient. This coefficient, defined as the probability of crossing the interface by a molecule impinging on it, was introduced by Knudsen [33] to reconcile the experimental findings with the predictions of the kinetic theory of gases. The experimentally observed evaporation rate in the ballistic regime is never greater than theoretically allowed by the kinetic theory. Though conceptually seemingly simple, the value of this coefficient turned out to be rather elusive [5, 34, 29, 35]. In view of that some authors call for major changes to the concept $[6,26,1,8]$.

Nonetheless, it is possible to combine the diffusive and kinetic descriptions into a single equation. It is customarily done by assuming that only at the distance $\Delta_{\mathrm{C}}$ from the droplet both descriptions are valid simultaneously (compare e.g. [36, 31]). This distance is comparable with the mean free path of molecules of ambient gaseous medium $l_{\mathrm{a}}$ but, in fact, the quantity has no clear physical meaning. The effective diffusion coefficient in Eq. (1) takes then the form

$$
D_{k}(a)=\frac{D}{a /\left(a+\Delta_{\mathrm{C}}\right)+4 D /\left(a \alpha_{\mathrm{C}} v\left(T_{\mathrm{L}}\right)\right)} .
$$

Similarly, the effective thermal conductivity of ambient gas may be expressed as

$$
\lambda_{k}(a)=\frac{\lambda}{a /\left(a+\Delta_{\mathrm{T}}\right)+4 \lambda /\left(a \alpha_{\mathrm{T}} \rho c_{P} v_{\mathrm{a}}\left(T_{\mathrm{L}}\right)\right)},
$$

where $\rho, c_{P}$ and $v_{\mathrm{a}}$ are density, specific heat capacity under constant pressure, and the average thermal velocity of molecules of ambient gas. $\Delta_{\mathrm{T}}$ is an analog of $\Delta_{\mathrm{C}}$ and $\alpha_{\mathrm{T}}$ is the thermal accommodation coefficient (compare [33]). Under standard temperature and pressure (STP) conditions $\Delta_{\mathrm{C}}, \Delta_{\mathrm{T}} \ll a$ and can be neglected for micron-sized droplets. It can be also easily noticed that kinetic effects, described with formulae 7 and 8, manifest only for relatively small droplets. The departure from the "radius-square-law" can be plainly visible only for $a \ll 4 D /\left(\alpha_{\mathrm{C}} v\left(T_{\mathrm{L}}\right)\right)$, which is usually well below $1 \mu \mathrm{m}$. An example of such departure that we attribute solely to kinetic effects (compare next section) is shown in Fig. 3, where our experimental results for a small triethylene glycol droplet are presented. In many cases it may be difficult to notice the manifestation of kinetic effects for 
data presented in $a^{2}(t)$ form. However, if the experimental $a(t)$ dependence is sufficiently smooth a different representation is more efficient. If Eqs. (1) and (3) are not strongly interconnected via temperature-dependent quantities, they can be both rewritten in a very convenient, linear form of

$$
\frac{1}{\dot{a}}=P_{1} a+P_{2},
$$

( $P_{1}$ and $P_{2}$ being composite constants) which encompasses kinetic effects and can supersede the "radius-square-law" (see inset in Fig. 4).

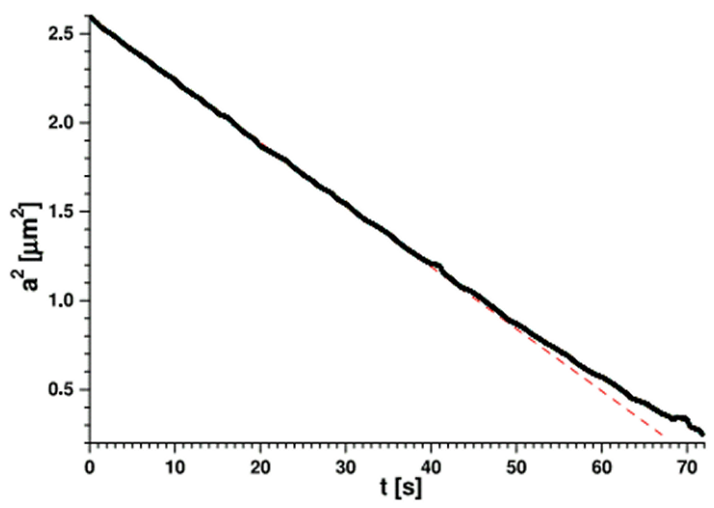

Fig. 3. Temporal evolution of the square of the radius of an evaporating droplet of triethylene glycol (TEG) droplet in $\mathrm{N}_{2}$ atmosphere initially void of TEG vapour $\left(\rho_{\mathrm{cc}}=0\right), T_{\mathrm{cc}}=298 \mathrm{~K}, 987 \mathrm{hPa}$ pressure. A straight, dashed (red) eye-guiding line was added to demonstrate the departure from the "radius-square-law".

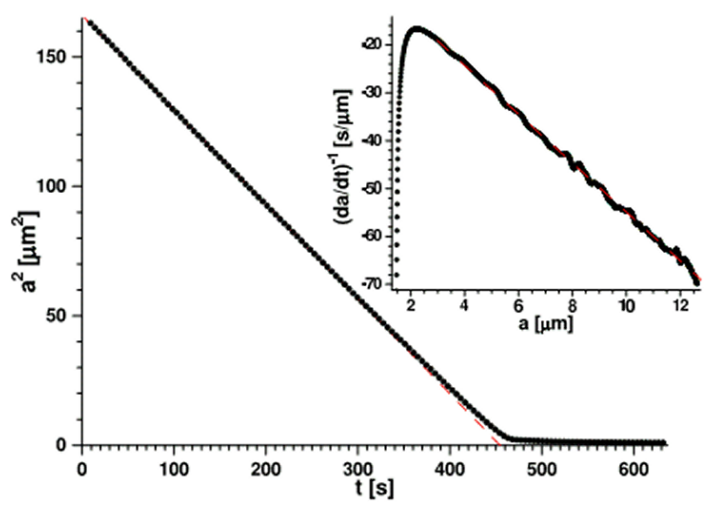

Fig. 4. Temporal evolution of the radius of an evaporating diethylene glycol (DEG) droplet in $\mathrm{N}_{2}$ atmosphere initially void of DEG vapour $\left(\rho_{\mathrm{cc}}=0\right), T_{\mathrm{cc}}=$ $298 \mathrm{~K}$, standard pressure. Evolution presented in $a^{2}(t)$ form (every fifteenth experimental point) and in $1 / \dot{a}(a)$ form in the inset (all experimental points). Dashed (red) lines correspond to linear fits for: $t<300 \mathrm{~s}$ in main figure and $3<a<12.5 \mu \mathrm{m}$ in the inset.

It is worth noticing that the evaporation into the atmosphere (initially) void of vapour stays isothermal even when the kinetic effects manifest for small droplets.
Equations (1) and (3) for $\rho_{\text {cc }}=0$ can be combined into

$$
\Delta T=\frac{D_{k}}{\lambda_{k}} h_{\mathrm{LV}} \rho_{\mathrm{sur}}\left(T_{\mathrm{L}}\right) .
$$

Then, if $\rho_{\text {sur }}\left(T_{\mathrm{L}}\right) \approx \rho_{\text {sat }}\left(T_{\mathrm{L}}\right)$ and $\Delta T \ll T_{\mathrm{cc}}, T_{\mathrm{L}}$, the Clausius-Clapeyron equation may be employed. If, further on, $h_{\mathrm{LV}} M \Delta T /\left(R T_{\mathrm{cc}} T_{\mathrm{L}}\right)$ is small, $\rho_{\mathrm{sat}}\left(T_{\mathrm{L}}\right)$ can be expressed in a simple form

$$
\rho_{\text {sat }}\left(T_{\mathrm{L}}\right) \approx \rho_{\mathrm{sat}}\left(T_{\mathrm{cc}}\right)\left(1+\frac{h_{\mathrm{LV}} M}{R} \frac{\Delta T}{T_{\mathrm{cc}}^{2}}\right) .
$$

Combining Eqs. (10) and (11) allows to disentangle $\Delta T$ :

$$
\frac{1}{\Delta T}=-\frac{\lambda_{k}}{D_{k}} \frac{1}{h_{\mathrm{LV}} \rho_{\mathrm{sat}}\left(T_{\mathrm{cc}}\right)}-\frac{h_{\mathrm{LV}} M}{R T_{\mathrm{cc}}^{2}} .
$$

Now, from Eqs. (7) and (8), it can be seen that the temporal evolution of $\Delta T$ originates from $\lambda_{k} / D_{k}$ only. Since $\lambda_{k}(a) / D_{k}(a)$ is always nearly constant so is $\Delta T$. Under the assumption of $\Delta_{\mathrm{C}}, \Delta_{\mathrm{T}} \ll a$ the details of this weak variability can be easily seen

$$
\frac{\lambda_{k}(a)}{D_{k}(a)}=\frac{\lambda}{D} \frac{a+4 D /\left(v \alpha_{\mathrm{C}}\right)}{a+4 \lambda /\left(v_{\mathrm{a}} \alpha_{\mathrm{T}} \rho c_{P}\right)} .
$$

Now the convex/concave behaviour of this expression depends on the proportion of

$$
\frac{4 \lambda /\left(v_{\mathrm{a}} \alpha_{\mathrm{T}} \rho c_{P}\right)}{4 D /\left(v \alpha_{\mathrm{C}}\right)} \approx \frac{\lambda}{c_{P} \rho D}=\text { Le, }
$$

where Le is the Lewis number. When the transfer of heat is more efficient than the transport of mass (Le $>1$ ) $\Delta T(a)$ is convex, otherwise $(\mathrm{Le}<1) \Delta T(a)$ is concave. These effects are, however, very minute, and seem to be of little practical significance.

\subsection{The influence of impurities}

A dramatic departure from the "radius-square-law" may ensue from the influence of impurities. Since the experimentally accessible liquids are never ideally pure, some non-linearity in $a^{2}(t)$ may always be anticipated, especially for evaporation leading to significant increase of impurities concentration. An example of such evaporation of diethylene glycol droplet, found in our experiments, is presented in Fig. 4. The departure from "radius-square-law" for $t>300 \mathrm{~s}$ in main figure and from equation 9 for $a<3 \mu \mathrm{m}$ (corresponding to $t>440 \mathrm{~s}$ in main figure) in inset is clearly visible. This departure for smaller radii makes an example of cooperation of kinetic effects and the influence of impurities (via Raoult's law). It turns out that this ambiguity can be resolved only partially even with 9 representation.

The influence of ideally soluble, non-surface-active, non-volatile impurity can be modelled in the simplest way by expressing $\rho_{\text {sur }}$ with the (simplified) Köhler equation $[37,31]$ :

$$
\rho_{\text {sur }}\left(T_{\mathrm{L}}\right)=\rho_{\text {sat }}\left(T_{\mathrm{L}}\right) \exp \left(\frac{-n_{s}}{\left(a / a_{0}\right)^{3}-n_{s}}\right),
$$

where $\rho_{\text {sat }}\left(T_{\mathrm{L}}\right)$ is the saturated (equilibrium) vapour density corresponding to temperature $T_{\mathrm{L}}, a_{0}$ is the initial droplet radius and $n_{s}$ is approximately equal to the initial mass fraction of impurities. Again, if necessary Eqs. (2) and (15) can be combined. 
In order to investigate the entanglement of the kinetic effects with the influence of impurities, we first performed (forward) numerical simulations using Eqs. (1), (3), (7) and $(8)$ together with $(15)$ to imitate the evaporation of a water droplet into dry nitrogen $\left(\rho_{\mathrm{cc}}=0\right.$, standard temperature and pressure (STP) conditions) versus the contents of impurities (represented by $n_{s}$ ). The dependence of quantities and parameters upon temperature was meticulously taken into account. We assumed $\alpha_{\mathrm{T}}=0.15$ and $\alpha_{\mathrm{C}}=0.14$. The simulated $1 / \dot{a}(a)$ for 3 values of $n_{s}$ are presented in Fig. 5 .

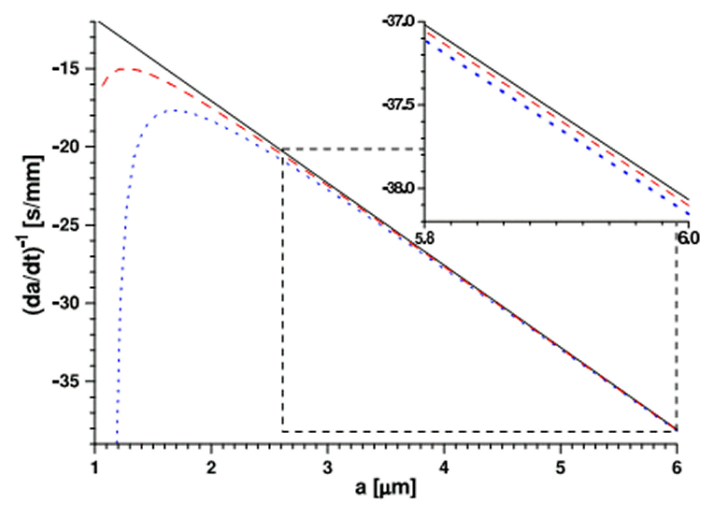

Fig. 5. Simulation of the effect of impurities upon droplet radius temporal evolution for water droplets evaporating into dry $\mathrm{N}_{2}$ atmosphere under STP conditions. Evolution shown in $1 / \dot{a}(a)$ representation. $n_{s}$ is proportional to the initial mass fraction of impurities. Solid (black) line: no impurities, $n_{s}=0$; dashed (red) line: $n_{s}=0.002$; dotted (blue) line: $n_{s}=0.005$. The region of apparently linear evolution is boxed. A small portion of this region is magnified in inset.

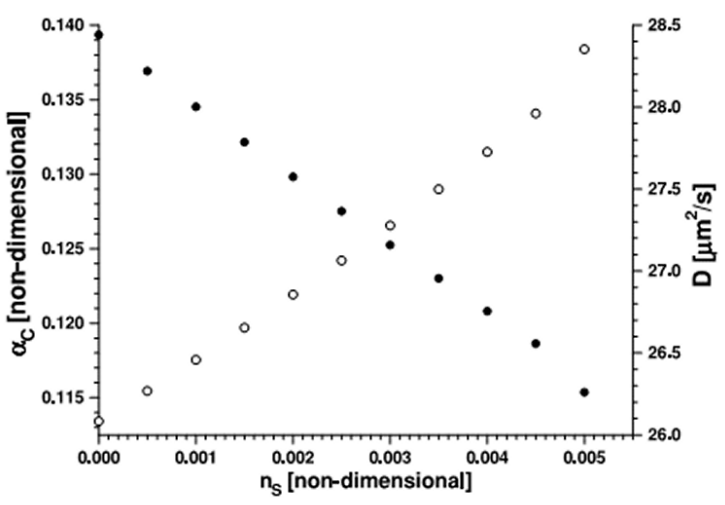

Fig. 6. The values of $D$ (open circles) and $\alpha_{\mathrm{C}}$ (solid dots) retrieved by fitting Eq. (9) (neglecting the Köhler equation) to the data from simulations (exemplified in Fig. 5) versus $n_{s}$ (impurities concentration) used in simulation. It can be seen that neglecting the effect of impurities in data analysis leads to significant errors.

The concentration of impurities grows as the droplet evaporates, and for high concentration the influence of impurities is clearly visible as highly non-linear region (see especially $1 / \dot{a}$ representation). Though this region can be easily isolated and excluded from consideration, the influence of impurities in seemingly linear region (box and inset in Fig. 5) can be easily overlooked, especially in view of experimental uncertainties and noise. Only when studied versus impurities initial concentration, it can be noticed that the higher $n_{s}$ the slower the evolution, even in seemingly unaffected region. Moreover, if we try to retrieve $D$ and $\alpha_{\mathrm{C}}$ from the simulation data by simply fitting (9) (neglecting the Köhler equation) it turns out that the retrieved values are much different from those supplied for the simulation (see Fig. 6). Even small, practically unavoidable amount of impurities initially present in the liquid, introduces error of several percent! Avoiding the problem requires utilising liquids of ultra-high purity, which is possible only in limited cases, e.g. for water. The disambiguation of the kinetic effects from the influence of impurities requires introducing a model describing the influence of impurities. Due to usually diverse and not exactly known type of impurities adopting a proper model may be a difficult task.

\subsection{Non-isothermal evaporation with additional heat source}

The last example of evolution disobeying the "radius-square-law", we would like to present, is the non-isothermal evaporation with additional source of heat. This mode of evaporation is encountered e.g. in burning fuel droplets and in droplets absorbing radiation (e.g. atmospheric aerosols, see e.g. [38]). An example of the later is presented in Fig. 7. A droplet of $\mathrm{C}_{60}$ fullerene nanocrystallite suspension in water was exposed to laser radiation and thus mildly heated.

As it was discussed in Sect. 5.2, if the suspension was non-absorbing, we would expect evolution slowing-down in respect of pure liquid (dashed (red) versus solid (green), radius-square-law-obeying line in Fig. 7) because of dispersed phase concentration change (Raoult's law) and/or surface blocking caused by scavenging of the dispersed phase by moving interface. In case of light absorbing suspension, first we observe evaporation speed-up in respect of the "radius-square-law", which indicates that the effect of heating prevails over the effects of suspension concentration change. At the later stage we indeed observe a rapid evaporation slow-down due to dispersed phase concentration change. The specific information carried by the departure from "radius-square-law", can still be recovered in case of evaporation with additional source of heat. However, it requires further extensions to the Maxwellian framework, which is beyond the scope of this work. As the result of the entanglement between the effects of dispersed phase concentration change and of temperature change, both transport equations would have to be used simultaneously. Though the mass transport equation remains seemingly simple, the heat balance becomes quite complicated (and temperature change may be high). We leave the detailed discussion of this issue for future works. 


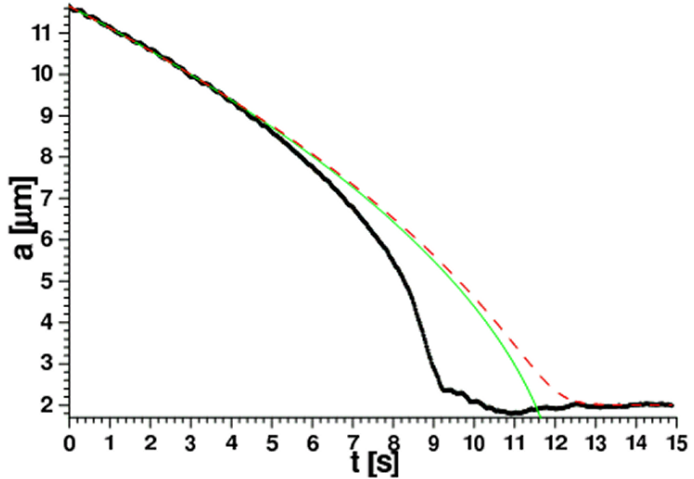

Fig. 7. Black dots: The temporal evolution of the radius of a droplet of suspension of $\mathrm{C}_{60}$ fullerenes nanocrystallites in water, evaporating in humid air $(R H>0.9), T_{\mathrm{cc}}=288 \mathrm{~K}$ at atmospheric pressure. Solid (green) and dashed (red) lines: evaporation of a droplet of pure water and of water with non-absorbing impurities respectively, calculated with the model for the above parameters.

\section{Non-isothermal evolution of small droplets - the persistence of the "radius-square-law"}

A very interesting case of rather unexpected adherence to the "radius-square-law" is encountered for very small droplets quickly evolving in nearly saturated atmosphere. In view of considerations from Sect. 5.1, we would rather expect a non-linearity introduced to the "radius-square-law" by the kinetic effects then. The most convincing are the experimental results of the University of Vienna/ University of Helsinki (UV/UH) group (see lower graph in Fig. 8 and e.g. [39-41, 36]) obtained for droplets growing in an atmosphere mildly supersaturated with vapour. However, similar effect seems to be present for nanoscopic droplets of the Lennard-Jones liquid evaporating into its own vapour (compare Figs. 3 and 4 in [6]). Our own measurements of moderately fast evaporation of water droplets into wet $\mathrm{N}_{2}$ atmosphere (see upper part of Fig. 8) are not contradicting but are not conclusive since the droplets were too large.

It is possible to demonstrate with Maxwellian model (both analytically and with numerical simulations) that the kinetic effects do manifest in this case, though in an unexpected way. In order to do so, both equations of transport: of heat and of mass must be used. Additionally, when $\rho_{\mathrm{cc}} \simeq \rho_{\mathrm{sat}}$, using the Kelvin equation is indispensable. Similarly as in Sect. 5.1, expanding exp in both Kelvin and Clausius-Clapeyron equations enables disentangling $\Delta T$. Under the easily fulfilled condition of $\Delta T \ll T_{\text {cc }}, T_{\mathrm{L}}$ we get

$$
\Delta T=\frac{K C}{a(1-K B)},
$$

where

$$
K=-\rho_{\mathrm{sat}} h_{\mathrm{LV}} \frac{D_{k}}{\lambda_{k}}, \quad B=\frac{h_{\mathrm{LV}}}{R T_{\mathrm{cc}}^{2}}, \quad C=\frac{2 M \sigma}{R T_{\mathrm{cc}} \rho_{\mathrm{L}}}
$$

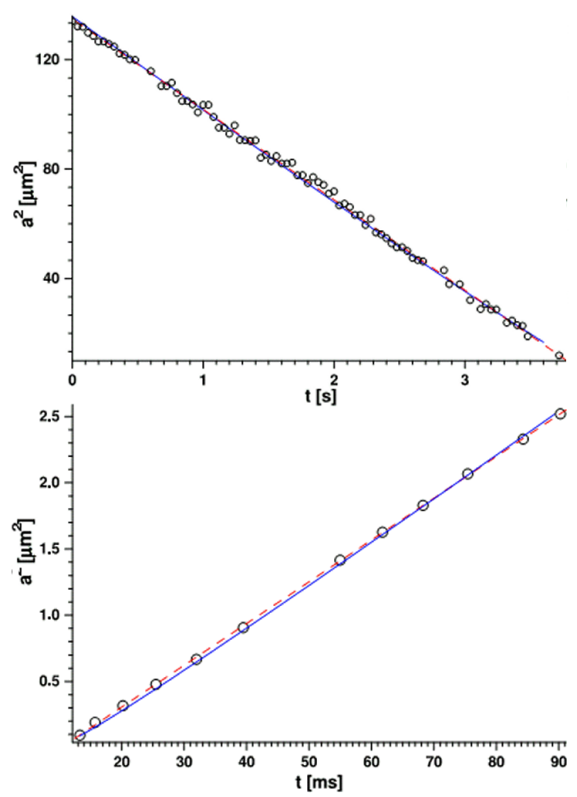

Fig. 8. Temporal evolution of droplet radii presented in $a^{2}(t)$ form. Upper part: moderately fast evaporation of a water droplet into wet $\mathrm{N}_{2}$ atmosphere $(R H>0.97)$, $T_{\mathrm{cc}}=298 \mathrm{~K}$, atmospheric pressure. Data from our experiments. Lower part: growth of water droplets condensing on silver nanoparticles in wet air $(R H=1.37)$ $T_{\mathrm{cc}}=268.2 \mathrm{~K}, 983 \mathrm{hPa}$ pressure. Data from "Ag03" set from [36]. Dashed (red) lines correspond to "radius-square-law", solid (blue) lines correspond to full model fits (no impurities).

are constants. The relative variability of $a$ is inherited by $\Delta T$. Thus, there appears a negative feedback via $\Delta T$. This is a rather unexpected finding that an evolution of a droplet near mechanical vapour-liquid equilibrium is non-isothermal while an evolution far from mechanical vapour-liquid equilibrium is. Employing Eq. (3) and formula (7) under the assumption of $\Delta_{\mathrm{C}}, \Delta_{\mathrm{T}} \ll a$ leads to the mass transport equation in the following form:

$$
\frac{\mathrm{d} a^{2}}{\mathrm{~d} t}=-\frac{F D}{a+4 D /\left(v \alpha_{\mathrm{C}}\right)},
$$

where

$$
F=\frac{2 \rho_{\text {sat }}}{\rho_{\mathrm{L}}} \frac{C}{1-K B} .
$$

It can be easily noticed that under the condition of $a \ll 4 D /\left(v \alpha_{\mathrm{C}}\right)$

$$
\frac{\mathrm{d} a^{2}}{\mathrm{~d} t}=-\frac{F}{4} v \alpha_{\mathrm{C}}
$$

It explains why the "radius-square-law" is valid under the considered conditions. However, the proportionality coefficient is rather unexpected, since for evaporation of small droplets into dry atmosphere we find $\mathrm{d} a / \mathrm{d} t \sim \alpha_{\mathrm{C}}$. The whole finding gives a strong impression that the existing description is more complicated than the process following a simple "radius-square-law".

While the kinetic effects visibly dominate, the evaporation/condensation coefficient $\alpha_{\mathrm{C}}$ can be measured. 
This is certainly the case for moderately fast evaporating water droplets in our measurements (see upper graph in Fig. 8), though the effect is obscured as the droplets are not very small. It is, however, fundamentally true for much faster evolutions of even smaller droplets from UV/UH group experiments (lower graph in Fig. 8). Adopting the models discussed in this work to nanoscopic droplets of Ref. [6] and finding the evaporation coefficient there requires much effort and we intend to present it in a separate paper.

In both graphs in Fig. 8 we present linear fits corresponding to the "radius-square-law" as well as numerical simulations performed with the unabridged model. For UV/UH experiment ("Ag03" set) we actually found the value of $\alpha_{\mathrm{C}} \approx 0.5$, which is by a factor of 2 smaller than proposed by UV/UH group and by a factor of 2 larger than proposed by Boston College/Aerodyne Research Inc. group [39]. Too high value of $\alpha_{\mathrm{C}}$ may follow from too small value of $h_{\mathrm{LV}}$ taken. Enthalpy of evaporation, playing a vital role in the heat transport equation, is defined and measured under equilibrium conditions and may be inadequate for an evolution far from thermal equilibrium. However, a proper correction to $h_{\mathrm{LV}}$ seems not to be known.

\section{Discussion and conclusions}

We have revisited the "radius-square-law" for evaporation of micro-droplets of non-ideal liquids and suspensions. We briefly discussed standard issues and concentrated on departures from the "radius-square-law" as well as on unexpected applicability case. To our knowledge, the last two issues were not discussed in the literature in a manner we propose, and we hope our findings may be of some help.

The temporal evolution of the square of droplet radius is linear primarily for large droplets of pure liquids. The vapour diffusion coefficient $D$ plays then the role of proportionality factor for this "radius-square-law": $\mathrm{d} a^{2} / \mathrm{d} t \sim D$. The evolution is isothermal then: the droplet temperature drop $\Delta T$ is constant though not equal to zero. The apparent linearity of $a^{2}(t)$ is retained even for relatively small droplets and relatively fast evaporation/condensation, since the $a^{2}(t)$ form is not very sensitive to kinetic effects. The linear form of $1 / \dot{a}(a)$, encompassing kinetic effects, is more sensitive and convenient, but requires very smooth $a(t)$ dependence.

For evaporation into atmosphere (initially) void of vapour the process continues to be isothermal. Though $\Delta T$ can be significant, its temporal evolution is usually very minute. On the other hand, the evolution of very small droplets in an atmosphere nearly saturated with vapour is non-isothermal. Then, the persistence of the "radius-square-law" is caused by coexistence of the kinetic effects and the strong effects of surface tension. Strangely, the evaporation coefficient $\alpha_{\mathrm{C}}$ replaces $D$ as proportionality factor for the "radius-square-law" then: $\mathrm{d} a^{2} / \mathrm{d} t \sim \alpha_{\mathrm{C}}$, while in dry atmosphere small droplets evolution do not obey the "radius-square-law": $\mathrm{d} a / \mathrm{d} t \sim \alpha_{\mathrm{C}}$. The intriguing fact, that the "radius-square-law" holds for very small droplets and fast evaporation/ condensation can be perceived just as a specific case of a more complex model. However, simple experimental results call rather for a simple description, which is still being sought.

Serious departures from the "radius-square-law" (or its derivatives) may be caused e.g. by the influence of impurities encountered in non-ideal liquids, by the kinetic and surface tension effects encountered for small droplets or by thermal imbalance encountered in light-absorbing droplets. We adhere to the opinion that in all such cases it is still possible to extract useful information from $a(t)$ dependence, though much care must be taken. Most remarkably, the values of thermodynamical parameters (e.g. diffusion and evaporation/condensation coefficients) found from the $a(t)$ dependence may be severely influenced by the effects of impurities, if unaccounted for. This fact seems to be quite often overlooked. Precise accounting for the effects of impurities may be difficult, however simple introducing of the Köhler equation yields a good approximation.

\section{Acknowledgments}

This work was supported by the Ministry of Science and Higher Education/European Science Foundation (ESF/PESC EPSD program) as a scientific project 2010-2013 and by Polish Ministry of Science and Higher Education under grant No. N N202 126837.

\section{References}

[1] F. Duan, I. Thompson, C.A. Ward, J. Phys. Chem. B 112, 8605 (2008).

[2] R. Hołyst, M. Litniewski, J. Chem. Phys. 130, 074707 (2009).

[3] W.A. Sirignano, Fluid Dynamics and Transport of Droplets and Sprays, Cambridge University Press, Cambridge 2010.

[4] N.A. Fuchs, Evaporation and Droplet Growth in Gaseous Media, Pergamon, London 1959.

[5] G.M. Nathanson, P. Davidovits, D.R. Worsnop, C.E. Kolb, J. Phys. Chem. 100, 13007 (1996).

[6] R. Hołyst, M. Litniewski, Phys. Rev. Lett. 100 , 055701 (2008).

[7] T. Ishiyama, T. Yano, S. Fujikawa, Phys. Fluids 16 , 2899 (2004).

[8] A.J.H. McGaughey, C.A. Ward, J. Appl. Phys. 91, 6406 (2002).

[9] A.H. Persad, C.A. Ward, J. Chem. Phys. B 114, 6107 (2010).

[10] V. Babin, R. Hołyst, J. Phys. Chem. B 109, 11367 (2005).

[11] V. Babin, R. Hołyst, J. Chem. Phys. 122, 024713 (2005).

[12] D. Jakubczyk, G. Derkachov, T. Do Duc, K. Kolwas, M. Kolwas, J. Phys. Chem. A 114, 3483 (2010). 
[13] M. Zientara, D. Jakubczyk, K. Kolwas, M. Kolwas, J. Phys. Chem. A 112, 5152 (2008).

[14] D. Jakubczyk, G. Derkachov, W. Bazhan, E. Łusakowska, K. Kolwas, M. Kolwas, J. Phys. D 37, 2918 (2004).

[15] F.G. Major, V.N. Gheorghe, G. Werth, Charged Particle Traps, Springer, Berlin 2005.

[16] E.J. Davis, M.F. Buehler, T.L. Ward, Rev. Sci. Instrum. 61, 1281 (1990).

[17] S. Arnold, Rev. Sci. Instrum. 62, 3025 (1991).

[18] R.A. Shaw, D. Lamb, J. Chem. Phys. 111, 10659 (1999).

[19] E.E. Allison, B.R.F. Kendall, Rev. Sci. Instrum. 67, 3806 (1996).

[20] H. Ulmke, T. Wriedt, K. Bauckhage, Chem. Eng. Technol. 24, 265 (2001).

[21] E.R. Lee, M.L. Perl, Universal Fluid Droplet Ejector, 1999, U.S. Pat. No. 5943075.

[22] S. Dehaeck, J.P.A.J. van Beeck, Exp. Fluids 45, 823 (2008).

[23] B. Steiner, B. Berge, R. Gausmann, J. Rohmann, E. Rühl, Appl. Opt. 38, 1523 (1999).

[24] N. Riefler, R. Schuh, T. Wriedt, Meas. Sci. Technol. 18, 2209 (2007).

[25] J.C. Maxwell, Collected Scientific Papers 11, 625 (1890).

[26] C.A. Ward, G. Fang, Phys. Rev. E 59, 429 (1999).

[27] J.O. Hirschfelder, C.F. Curtiss, R.B. Bird, Molecular Theory of Gases and Liquids, Wiley, New York 1954.

[28] J.D. Smith, C.D. Cappa, W.S. Drisdell, R.C. Cohen, R.J. Saykally, J. Am. Chem. Soc. 128, 12892 (2006).

[29] W.S. Drisdell, C.D. Cappa, J.D. Smith, R.J. Saykally, R.C. Cohen, Atmos. Chem. Phys. 8, 6699 (2008).
[30] A.M. Moyle, P.M. Smidansky, D. Lamb, in: Am. Meteorological Soc. Proc. 12th Conf. Cloud Physics, Madison (WI) 2006, https:// ams. confex.com/ams/pdfpapers/111916.pdf .

[31] H.R. Pruppacher, J.D. Klett, Microphysics of Clouds and Precipitation, Kluwer, Dordrecht 1997.

[32] G. Fang, C.A. Ward, Phys. Rev. E 59, 417 (1999).

[33] M. Knudsen, The Kinetic Theory of Gases: Some Modern Aspects, Methuen, London 1950.

[34] P.A.J. Bagot, C. Waring, M.L. Costen, K.G. McKendrick, J. Phys. Chem. C 112, 10868 (2008).

[35] F.R. McFeely, G.A. Somorjai, J. Phys. Chem. $\mathbf{7 6}$, 914 (1972).

[36] P.M. Winkler, A. Vrtala, P.E. Wagner, M. Kulmala, K.E.J Lehtinen, T. Vesala, J. Geophys. Res. 111, D19202 (2006)

[37] S.K. Friedlander, Smoke, Dust and Haze Fundamentals of Aerosol Dynamics, Oxford University Press, New York 2000.

[38] C. Erlick, V. Ramaswamy, J. Geophys. Res. 108, 2963 (2003)

[39] P. Davidovits, D.R. Worsnop, J.T. Jayne, C.E. Kolb, P. Winkler, A. Vrtala, P.E. Wagner, M. Kulmala, K.E.J. Lehtinen, T. Vesala, M. Mozurkewich, Geophys. Res. Lett. 31, L22111 (2004).

[40] A. Laaksonen, T. Vesala, M. Kulmala, P.M. Winkler, P.E. Wagner, Atmos. Chem. Phys. Discuss. 4, 7281 (2004).

[41] P.M. Winkler, A. Vrtala, P.E. Wagner, M. Kulmala, K.E.J. Lehtinen, T. Vesala, Phys. Rev. Lett. $\mathbf{9 3}$ 75701 (2004). 